\title{
Detection efficiency of acoustic biotelemetry sensors on Wave Gliders
}

\author{
Megan Cimino ${ }^{1,2^{*}} \mathbb{0}$, Michael Cassen ${ }^{1}$, Sophia Merrifield ${ }^{1}$ and Eric Terrill ${ }^{1}$
}

\begin{abstract}
Background: Detecting tagged animals in coastal environments is often limited to stationary arrays of acoustic receivers that can decode transmissions from tags on animals. However, mobile autonomous platforms are becoming important tools that support the science of understanding biophysical relationships because they can concurrently detect tagged individuals and measure properties of their ocean habitat. To assess the effectiveness of these emerging platforms, proper validation and range detection studies are necessary. Here, we report on the deployment of a wave powered unmanned surface vessel, the Liquid Robotics Wave Glider SV3, equipped with a forward- and backward-facing acoustic receiver (VR2W) and transceiver (VR2Tx) at $4 \mathrm{~m}$ depth. Surveys were conducted around two stationary moorings equipped with receivers, transceivers or tags emitting signals with different power outputs.
\end{abstract}

Results: During our study, the sea state was mild with low wind speeds ( $<10$ kts) and small wave heights ( $<0.8 \mathrm{~m}$ ). We determined the influence of environmental and vehicle factors on the detection range of transmitters with various power outputs. Overall, the highest detection efficiencies $(\sim 50-95 \%)$ were at distances $<0.1 \mathrm{~km}$, lower detection efficiencies $(0.1-50 \%)$ were $>0.5 \mathrm{~km}$ and the maximum range was $0.5-1.2 \mathrm{~km}$. The forward-facing receiver had almost half the detection efficiency of the backward-facing transceiver, suggesting a backward configuration is optimal to reduce the influence of the moving platform. The higher power output transmitters had a $20 \%$ detection efficiency to ranges of $\sim 0.5 \mathrm{~km}(153 \mathrm{~dB}$ ) and $\sim 0.8 \mathrm{~km}(160 \mathrm{~dB})$. Distance between the receiver and transmitter was the main factor affecting detection probability, with background noise, receiver heading, angle between transmitter and receiver and wave height also being important. Wind speed, water temperature, mooring line tilt angle and vehicle dynamics were found not to be as important over the limited range of conditions over which our study was conducted.

Conclusions: Wave Gliders equipped with receivers can provide useful data and can be an effective biotelemetry asset that could supplement stationary arrays of acoustic receivers or act as an exploratory technology to search for biologically important areas.

Keywords: Wave Glider, Acoustic telemetry, VEMCO, Range testing, Detection efficiency

\section{Background}

Studies of the long-term temporal and spatial ecology and behavior of marine organisms have been augmented by acoustic telemetry [1]. Animals can be tagged with acoustic transmitters and their movements monitored by coastal arrays of stationary hydrophones or acoustic receivers. Information attained from passive telemetry studies provides insights far beyond what can be

\footnotetext{
*Correspondence: mecimino@ucsc.edu

${ }^{2}$ Institute of Marine Science, University of California Santa Cruz, SantaCruz, CA 95064, USA

Full list of author information is available at the end of the article
}

observed by eye, video or active tracking and is a critical component to conservation and management efforts [2]. However, records of animal locations are limited to areas that have receivers, typically shallow continental shelves, which often leave large gaps in our understanding of animal movements due to limited data coverage. With technological advances, there are a number of marine vehicles that are capable of tracking animals or detecting their presence, but in many cases, proper validation (e.g., range detection testing) is needed to interpret the acoustic data, to establish the utility of the method and to develop effective sampling plans [2]. 
Autonomous underwater vehicles (AUVs) or platforms are becoming more common telemetry assets because receivers can be self-contained and externally attached, or integrated for real-time detection while simultaneously measuring physical and biological ocean properties. For example, buoyancy-driven Slocum gliders with integrated VEMCO cabled receivers (VR2c) or externally mounted mobile transceivers (VMTs) have been used to study sturgeon and shark habitats [3-5]. Lotek stereo-hydrophone acoustic receiver systems have been integrated into propelled OceanServer IVER2 AUVs to track and follow leopard sharks [6, 7]. VEMCO receivers have been externally mounted and Lotek hydrophones integrated into a propeller-driven REMUS-100 AUV [8, 9]. A Liquid Robotics Wave Glider (WG) also allows for real-time detections of tagged marine life using an integrated VR2c [10]. These platforms provide exciting new capabilities to track, follow and visualize fish movements in near real time, but implementing AUV-supported telemetry is still in its infancy. One complication is that AUVs generate platform-specific acoustic noise that is sea-state dependent and additive to the ocean's ambient sound field, which also varies in time and space. The detection range of an acoustic receiver is dependent on the acoustic landscape, both the background noise and the propagation environment, as well as the receiver array configuration [2, 11-14]. Therefore, to ensure an AUV is an efficient telemetry asset, it is necessary to conduct range tests, have onboard processing for geolocation or use multiple vehicles for coordinated path planning based on received detections [6-8]. Propelled vehicles are aptly suited for the latter tasks but due to the high power cost of propelled motion, they are restricted by battery life to deployments often lasting $<1$ day. In comparison, buoyancy- or wave-driven vehicles can conduct continuous surveys for months and can adapt their location to stay within biologically interesting regions, which may migrate in space, in contrast to measurements from fixed arrays.

WGs are an emerging autonomous surface platform that is made up of a surface float and underwater sub. While WGs have successfully detected tagged animals $[10,15]$, it is unclear whether their detection efficiency is comparable to other more streamlined and faster vehicles. WGs are unique in that they have a surface and underwater expression, and are capable of measuring both air and sea conditions. They can be at sea for over a year and can transit long distances or station keep in one location, which in theory could allow the platform to act as a stationary receiver, an along or across shore gate, or even follow transoceanic migrations. Long deployments are especially useful for exploration into new areas where animal habitats are unknown. Receivers have been integrated into WGs, however is it expensive to do so, making knowledge of performance necessary to justify the cost. Therefore, in this study, we tested the detection efficiency of externally mounted receivers in two configurations (forward and backward facing) on the WG sub in relation to the listening environment to investigate the utility of this platform in future telemetry studies. We identified the factors that may influence detection ranges as this could inform the applicability of the technique.

\section{Results}

\section{Detection environment}

Over the one-week mission, the WG spent approximately 3 days in the vicinity of the north mooring and 4 days near the south mooring (Figs. 1, 2). All tags, receivers, and transceivers on the mooring lines were at a depth below the thermocline (as measured on February 2) while the depth of the sub was at the base of the thermocline near the north mooring and within the thermocline near the south mooring (Fig. 3). The vertical temperature and sound speed profiles were similar within and between regions (Fig. 3).

We found that noise levels varied over time and by location. A diel signal in noise was most evident in the southern mooring region on the high, medium and low transceivers (Fig. 4a). Low noise (very good to good) conditions are typically less than $300 \mathrm{mV}(\sim 5.2 \mathrm{~dB})$, moderate to challenging conditions are within $300-650 \mathrm{mV}$ $(\sim 5.2-11.9 \mathrm{~dB})$ and high noise conditions are 650$950 \mathrm{mV}(\sim 11.9-15.2 \mathrm{~dB})$ (VEMCO Ltd.). In the southern region, daytime noise was often around $2 \mathrm{~dB}$, whereas nighttime noise levels generally peaked between 5 and $11 \mathrm{~dB}$. The diel pattern in noise levels was greatest on February 3 and decreased over time. Noise on the WG transceiver was more consistent at $3.5 \mathrm{~dB}$. We found no diel noise pattern in the northern mooring region (as sampled by the WG), and noise levels on the WG closely matched noise levels recorded on mooring transceivers in the southern region. The difference between the minimum noise levels on the WG and the mooring line transceivers suggests the WG produces $\sim 2 \mathrm{~dB}$ of sound above the background noise as parameterized by the receiver.

Water temperature varied throughout the mission (Fig. 4b). A daily tidal signal on the mooring transceivers was seen, but it was less distinguished on the WG. A warming trend was seen from February 3-7, perhaps related to low wind and small waves, which dissipated after a breezy period on February 6-7. Overall, wind speeds were low $(<10 \mathrm{kts})$ and waves were small $(<0.8 \mathrm{~m})$ throughout the deployment (Fig. 4e).

Orientation of the transceivers and WG sub was measured by the transceivers themselves and an external three-axis accelerometer on the WG sub. A $0^{\circ}$ tilt 


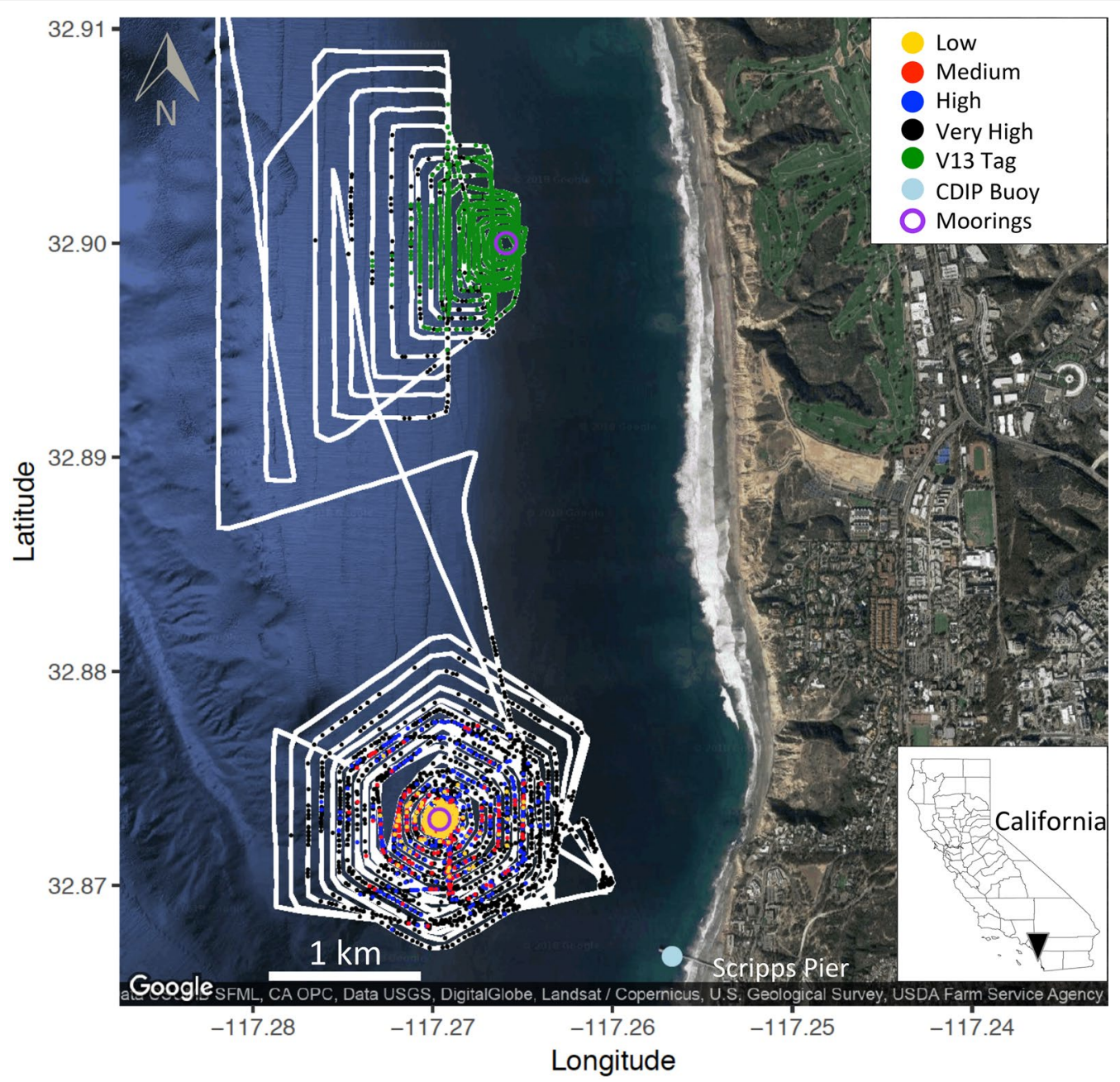

Fig. 1 Wave Glider path and location of acoustic detections off San Diego, CA. The Wave Glider mission centered around two separate moored lines equipped with transceivers or tags using different power outputs (high, medium and low) to determine the detection efficiency of a Wave Glider. A transceiver mounted on the Wave Glider (very high power output) was also heard by receivers/transceivers on the moored lines

measured by the transceivers indicates the transceiver is vertical with the hydrophone pointing toward the surface and $90^{\circ}$ is horizontal (Fig. 4c). Tilt angles on the medium and low transceiver were similar (Pearson $R=0.94, p<0.05$ ), an average of $25^{\circ}$ (ranging from $5^{\circ}$ to $\left.40^{\circ}\right)$ as the transceivers were mounted vertically on the mooring line (the high transceiver malfunctioned). This slight tilt is due to slack in the mooring line and likely related to wind, wave and tidal effects. Tilt angle on the WG transceiver was on average $81^{\circ}$ with a very small range $\left(78^{\circ}-88^{\circ}\right)$, as the receiver was mounted horizontal and facing backward. This tilt angle suggests the hydrophone was pointed slightly up toward the surface. This was confirmed by the accelerometer data, which showed the sub had an average downward pitch of $-10.5^{\circ}$ (ranging from $10.5^{\circ}$ to $-20.3^{\circ}$ ) and a small roll averaging $1.2^{\circ}$ (ranging from $-9.8^{\circ}$ to $11.8^{\circ}$ ) (Fig. $4 \mathrm{~d}$ ). The 3-minute running mean and standard deviation of the sub pitch revealed times with increased sub motion. We considered this standard deviation of the pitch to be indicative of the dynamics of the sub, which increased with higher winds and wave heights (Fig. 4e). Finally, there were few detections when the WG was far from the mooring lines (Figs. 1, 4f).

\section{Acoustic detections}

When the WG was within $1.3 \mathrm{~km}$ of the mooring lines, we determined the number of emitted acoustic signals and the number of decoded signals by each tag and transceiver/receiver, which differed between the forward- and backward-facing receiver/transceiver. During the north mooring survey, the V13 tag emitted 7144 coded acoustic 


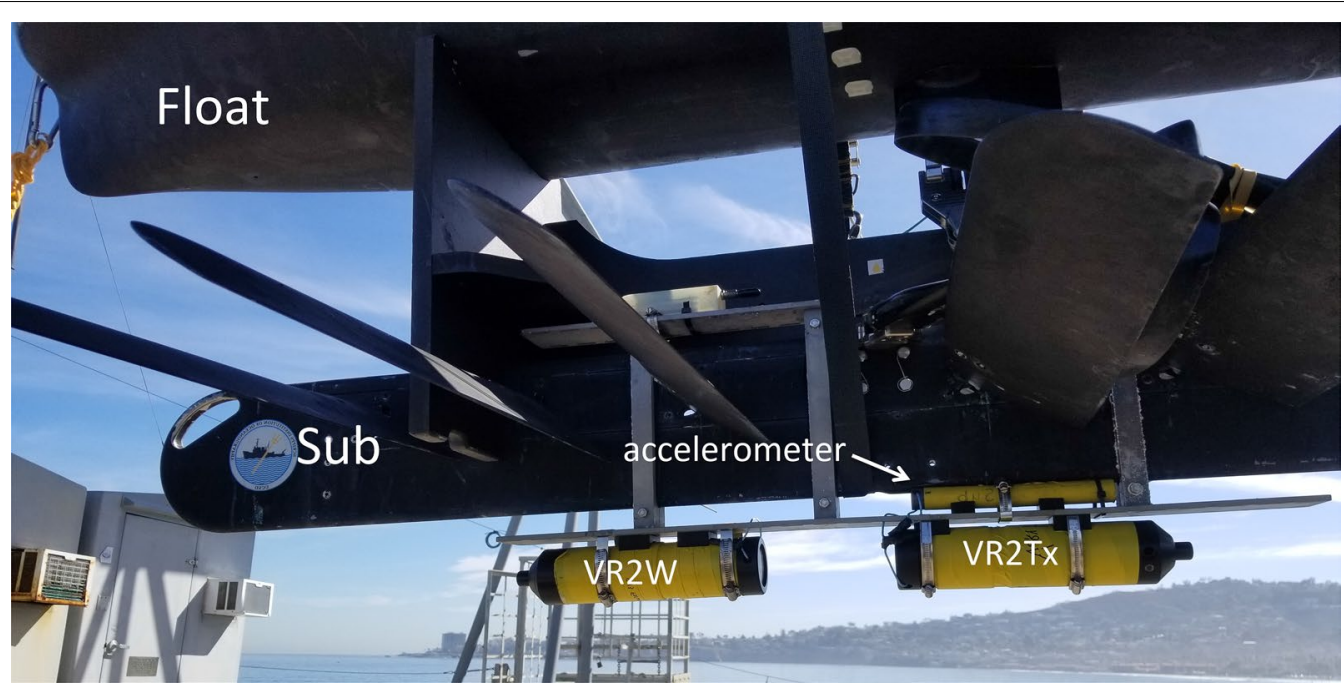

Fig. 2 Wave Glider SV3 consists of a float and sub connected by a tether. The surface float contains communications, solar panels, batteries and integrated sensors. The sub has wings, a rudder and thruster for propulsion. The photograph shows custom-made steel brackets and plates where the VR2W receiver (forward facing), VR2Tx transceiver (backward facing) and accelerometer were mounted. The front of the vehicle is on the left

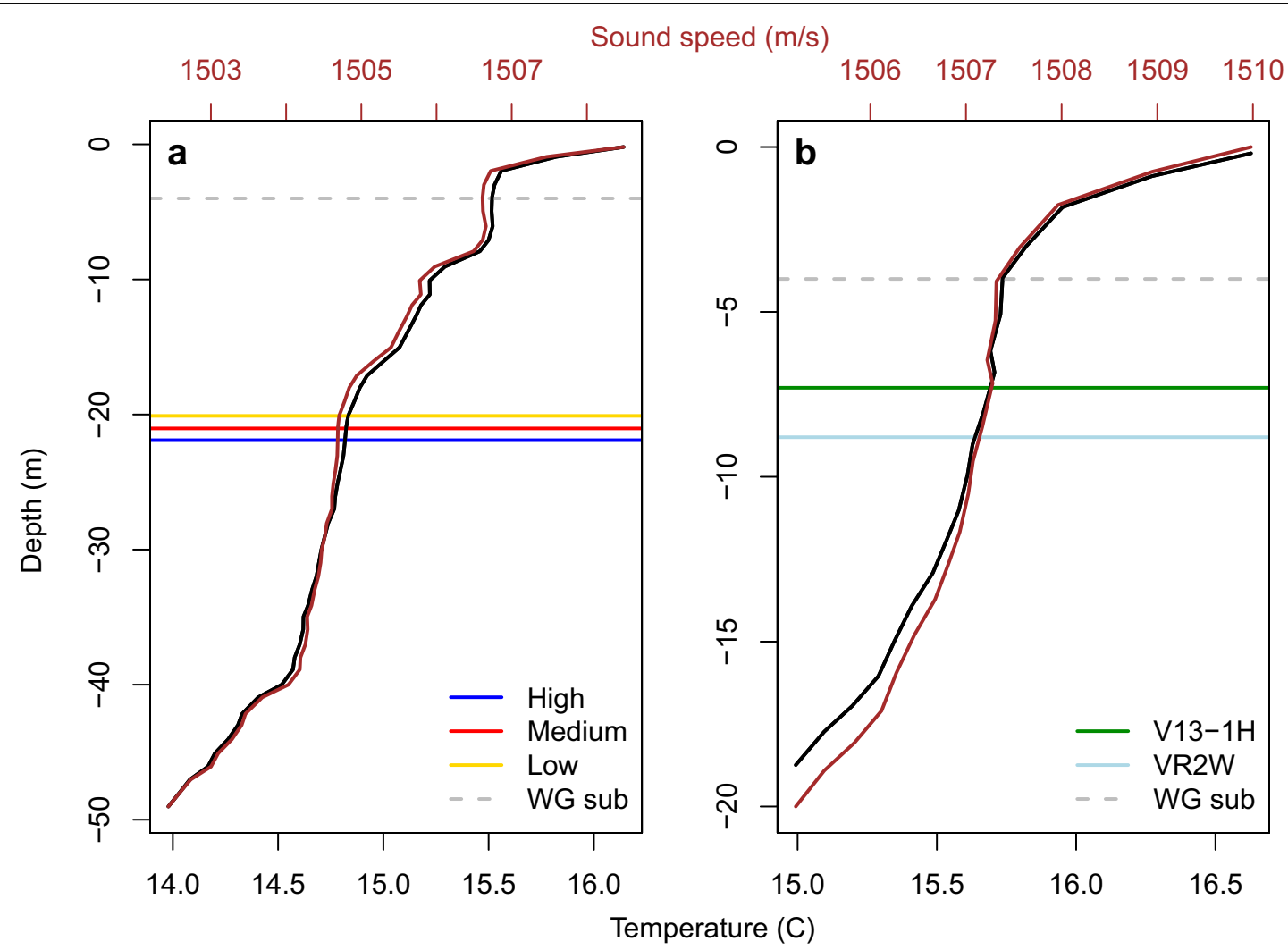

Fig. 3 Temperature and sound speed profiles at the $\mathbf{a}$ south deep mooring and $\mathbf{b}$ north shallow mooring. Temperature and sound speed profiles were measured on the day of deployment on February 2, 2018. Horizontal lines represent the depth of attached VR2Tx transceivers (high, medium and low power output), V13-1 H tag and VR2W receiver on the mooring lines. The depth of the Wave Glider (WG) sub with a transceiver (very high power output) and VR2W receiver is shown 


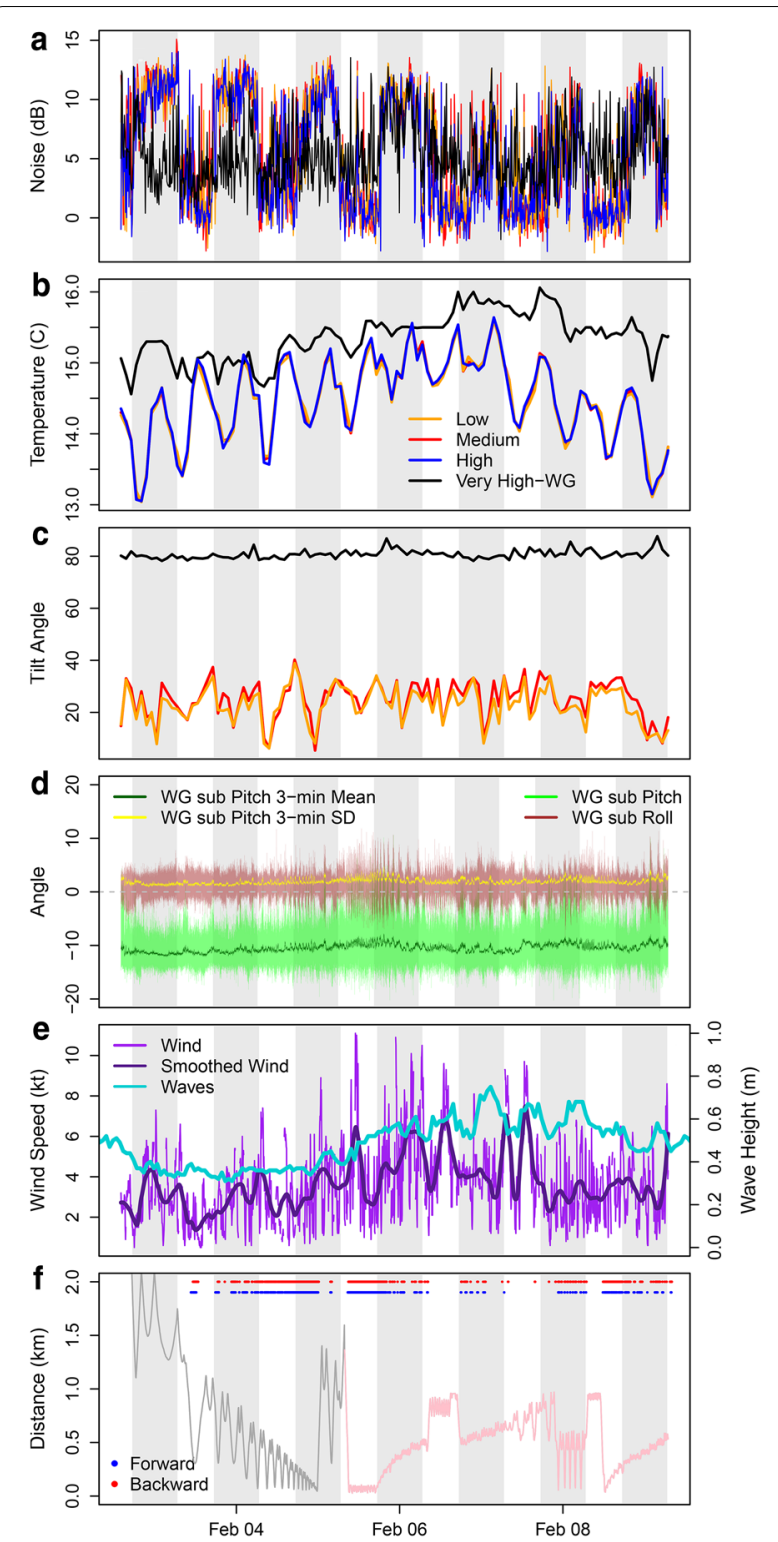

Fig. 4 Acoustic detections throughout the Wave Glider (WG) mission in relation to factors that may affect detection efficiency. a Noise, $\mathbf{b}$ water temperature and $\mathbf{c}$ tilt angle measured on the three transceivers (high, medium and low) on the south mooring line and the very high transceiver on the WG. $\mathbf{d}$ Pitch and roll angle of the WG sub and a running mean and standard deviation (SD, 3-minute time window) of the pitch. $\mathbf{e}$ Wind speed measured on the WG in 5-minute increments, smooth wind (Loess smoother, span $=0.05$ ) and significant wave height. $\mathbf{f}$ Distance between the WG and the north (gray) and south (pink) mooring, with detection times on the forward and backward receivers. Night periods (sunset to sunrise) are shaded gray

signals that were heard on the VR2W receiver attached to the same mooring line. The forward-facing receiver on the WG decoded 1098 detections (15.37\%), and the backward-facing transceiver decoded 2027 detections (28.37\%). Together, after removing double detection counts, the forward-facing receiver and backward-facing transceiver recorded 2334 detections (32.67\%). During the south mooring survey, the low, medium and high transceivers emitted 4032, 4039, and 4039 coded acoustic signals, respectively. The forward-facing receiver decoded 153 pings (3.79\%) from the low, 242 (5.01\%) from the medium and 334 (8.27\%) from the high transceiver, and the backward-facing transceiver decoded 240 (5.95\%) from the low, 385 (9.53\%) from the medium and $550(13.62 \%)$ from the high transceiver. Together, after removing double detection counts, the forward-facing receiver and backward-facing transceiver recorded 285 detections $(7.07 \%)$ from the low, 449 detections (11.11\%) from the medium and 650 detections (16.09\%) from the high.

The very high transceiver mounted on the WG was also emitting acoustic signals that could be heard by the mooring line transceivers and receivers. During the north mooring survey, the very high transceiver emitted 1908 signals and the receiver on the mooring line decoded 720 detections (37.73\%). During the south mooring survey, the very high transceiver emitted 4005 signals. On the mooring line, the low transceiver decoded 1512 pings (37.75\%), the medium transceiver decoded 1531 pings (38.22\%) and the high transceiver decoded 1177 pings (29.39\%). While the high, medium and low transceivers were mounted close together, the high transceiver (1-2 $\mathrm{m}$ deeper) decoded $~ 10 \%$ fewer pings compared to the low and medium transceivers, suggesting there could have been shading effects from the transceivers mounted above.

The detection efficiency varied by distance between the transmitter and receiver. The detection ranges decreased linearly with distance on a log scale (Fig. 5). The highest detection efficiencies ( 50-95\%) were at distances $<0.1 \mathrm{~km}$ (Fig. 5), much higher than $30-40 \%$ detection efficiencies for Slocum gliders [16]. On the WG, the forward-facing receiver had almost half the detection efficiency of the backward-facing transceiver (Fig. 5a, b). The V13 tag $(153 \mathrm{~dB})$ and high transceiver $(154 \mathrm{~dB})$ power levels were similar but we found a higher detection range of the V13 tag compared to the high transceiver on both the forward- and backward-facing receiver/transceiver on the WG. High, medium and low power outputs had higher to lower detection efficiencies, respectively (Fig. 5a, b). At distances $>0.5 \mathrm{~km}$, detections were sparse on the forward-facing receiver and backward-facing transceiver. In comparison, detection efficiencies of the very high transceiver mounted on the WG to the mooring line receivers/transceivers were higher (Fig. 5c). In general, there were similar efficiencies between receivers/ 

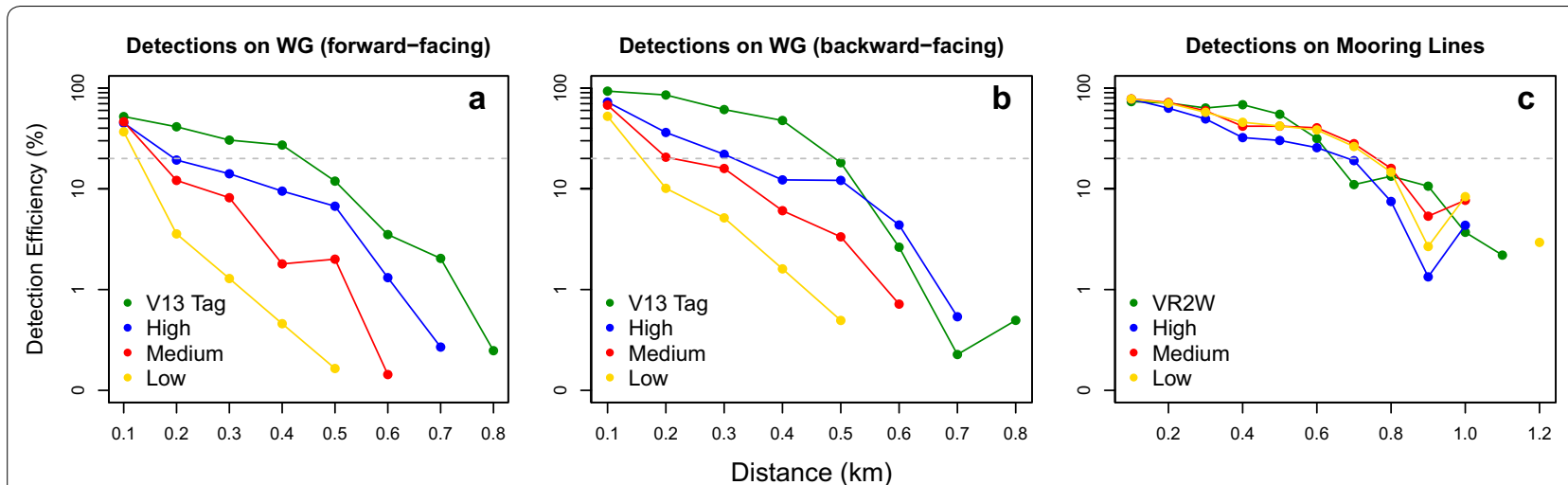

Fig. 5 Detection efficiency by distance between the Wave Glider (WG) and mooring line. Detection efficiency of a V13 tag and transceivers with different power outputs (high, medium and low) attached to a mooring line on $\mathbf{a}$ the forward-facing VR2W receiver and $\mathbf{b}$ the backward-facing VR2Tx transceiver mounted on the WG. c Detection efficiency of the WG transceiver (very high power output) on the mooring line VR2W receiver (shallow mooring) and three transceivers (high, medium and low; deep mooring). The $y$-axis is on a log scale. The $20 \%$ detection efficiency is shown by the gray dashed line

transceivers within each distance bin with slightly lower detection efficiencies on the high transceiver (Fig. 5c). Comparatively, the $20 \%$ detection efficiency of the forward-facing receiver was $\leq 0.4 \mathrm{~km}$, the backward-facing transceiver was $<0.5 \mathrm{~km}$ and the transceivers/receivers had a $20 \%$ detection efficiency of the very high transceiver to $\sim 0.8 \mathrm{~km}$. The maximum detection range on the backward- and forwarding-facing transceiver/receiver was $0.8 \mathrm{~km}$ while the maximum for the very high receiver was $1.2 \mathrm{~km}$.

\section{Environmental and vehicle predictors of acoustic detections}

We studied the factors that affected whether an acoustic ping was detected by each receiver/transceiver in the north and south mooring regions. We ran three suites of models to explain (1) detections on the backward-facing transceiver on the WG (model A-D), (2) detections on the forward-facing receiver on the WG (model E-H) and (3) detections on the WG's very high transceiver on the mooring lines (model I-L) (Table 2). The environmental parameters tested in each model included a combination of wind speed, wave height; sub noise, pitch, roll and dynamics; WG heading and angle; distance; and mooring noise, tilt and temperature (see Table 2).

Our boosted regression tree (BRT) models performed well with all models having an AUC $>0.80$ (Table 2). The explained deviance ranged from 23 to $64 \%$ depending on power output and transceiver/receiver location. Models explaining detections on the backward-facing transceiver (A-D) explained more of the deviance compared to the models explaining detections on the forward-facing receiver $(\mathrm{E}-\mathrm{H})$. For both forward and backward detections on the WG in the south (models A-C, E-G), the low transceiver models ( $C$ and $G$ ) explained more of the deviance ( 50\%) compared to medium (B and F, 45\%) and high (A and $\mathrm{E}, \sim 35 \%$ ). In the north region (models $\mathrm{D}$ and $\mathrm{H}$ ), detections of the V13 tag on the backward-facing transceiver had the highest explained deviance (D, 64\%) while detections on the forward-facing receiver were similar to the explained deviance of other models on that receiver $(\mathrm{H}, \sim 40 \%)$. The explained deviance was similar $(\sim 28 \%)$ for all models (I-L) investigating detections on mooring lines of the very high transceiver.

The five most important variables in explaining detection probability overall were distance, noise, angle between the WG and mooring (i.e., transmitter and receiver), WG heading and wave height (Additional file 1). Overwhelmingly, distance contributed the most to the models $(\sim 40-80 \%)$ and was the most importance predictor variable in all models. Noise, angle, heading and wave height contributed on average $6.1 \%$ (ranging from $2-12 \%), 6.0 \%$ (3-14\%), $4.2 \%(1-7 \%)$ and $4.1 \%(1-10 \%)$, respectively (Additional file 1 ). The remaining predictor variables, which included wind speed, sub pitch, roll and dynamics, temperature and mooring tilt, contributed on average $~ 2.9 \%$ (ranging from 0.6 to $6.7 \%$ ). For detections of the very high transceiver (model I-L), distance had a lower contribution comparatively, and WG factors (sub motion, noise) and orientation of the WG to the mooring line (angle, heading, tilt) were more important.

The relationship between the effects of each predictor variable on detections is shown in Fig. 6 and Additional file 2. Partial plots may not reveal the comprehensive effects of a predictor on detections when strong interactions between predictor variables are present (Additional file 3). Detections were more probable at short distances and low noise and decreased as distance and noise increased (Fig. 6a, b). Detection probability was higher at lower angles, where an angle of $0^{\circ}$ indicated the 

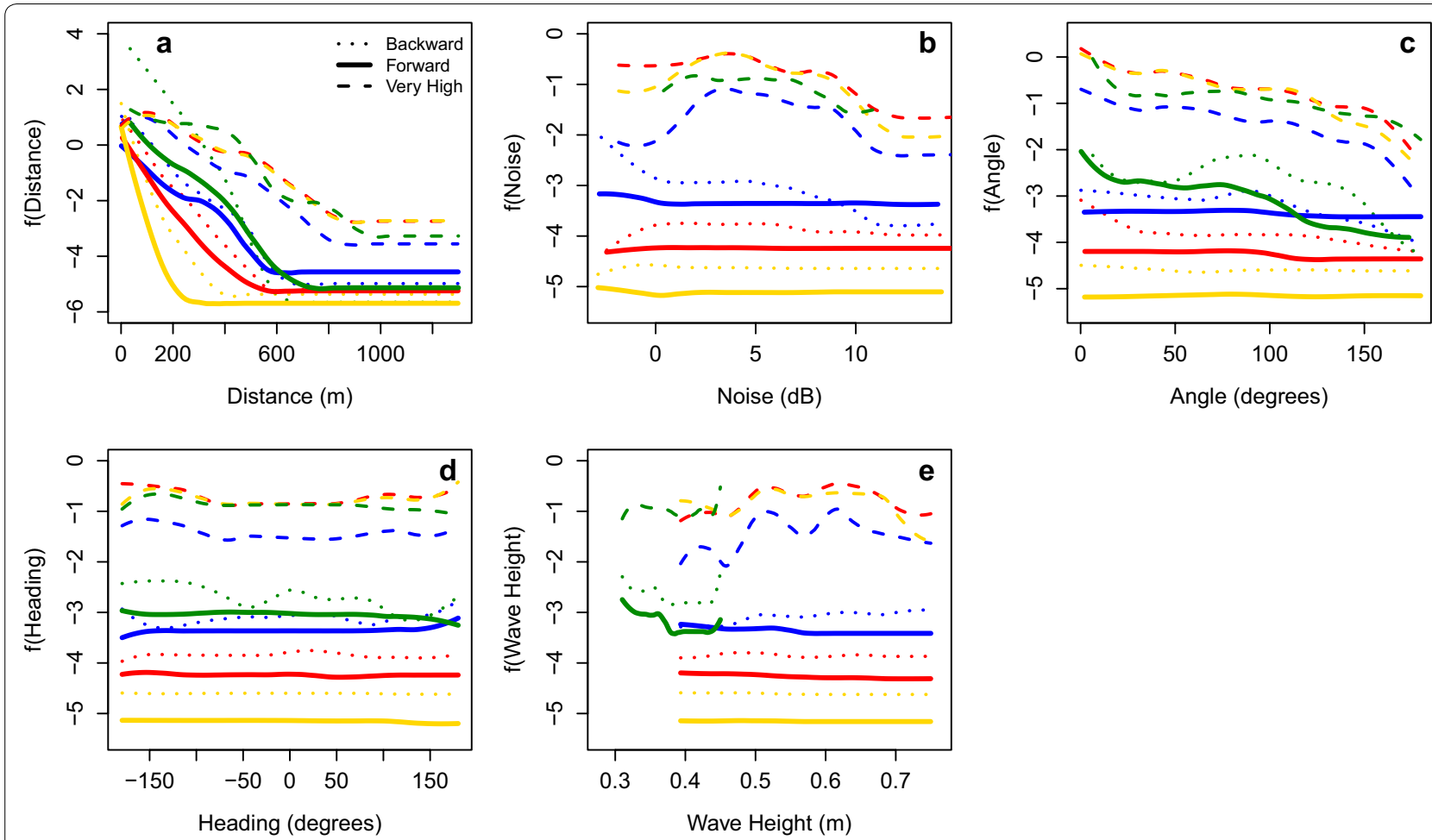

Fig. 6 Partial dependence plots of boosted regression trees (BRTs) relating acoustic detections (yes/no) to environmental and vehicle predictors. The five most important predictors include $\mathbf{a}$ distance between the Wave Glider (WG) and mooring line, $\mathbf{b}$ noise, $\mathbf{c}$ angle between the WG receiver/ transceiver and the mooring line, $\mathbf{d}$ WG heading and $\mathbf{e}$ wave height. Each line corresponds to a model in Table 2. Green lines represent the north mooring (V13 tag or receiver), and blue, red and yellow lines are the three transceivers (high, medium and low) on the mooring line. Backward and forward represent models that investigated drivers of acoustic detections on the WG transceiver/receiver. Very high represents models that investigated drivers of detections on the mooring lines from the very high transceiver mounted on the WG. These plots show the effect of a variable on the response after accounting for the average effects of other model variables

receiver/transceiver was pointing toward the mooring line and $180^{\circ}$ meant they were facing in opposite directions (Fig. 6c). In general, a south heading $\left(-180^{\circ} / 180^{\circ}\right)$ and small waves were positively related to detection probabilities (Fig. 6d, e). For variables of minor importance, the relationships varied but in general, a positive pitch improved detection probabilities for the backwardfacing transceiver and negative pitch improved detection probabilities for the forward-facing receiver, which result in the hydrophone pointing slightly downward. A positive relationship was found between detections and no roll, low wind speeds, little tilt in the mooring line (thus, transceivers are vertical) and lower sub dynamics (Additional file 2).

Interactions between predictor variables also helped explain detection probabilities. There were often interactions between distance and noise, angle and pitch (Additional file 3). All of these interactions showed that when the distance was small, there was little effect of noise, angle and pitch. However, as distance increased, these variables became more important in determining detection efficiencies (similar to [17]). For example, at distances greater than $\sim 200 \mathrm{~m}$ and noise $>\sim 0 \mathrm{~dB}$ or angles $>\sim 30^{\circ}$, the probability of detection dropped to about $50 \%$ (Fig. 7). This effect is explained by the signalto-noise ratio where the transmitted signal dominates the noise at close distances and conversely, the transmitted signal is lost due to attenuation or interference at far distances.

\section{Discussion}

In testing the detection range of receivers mounted onto a WG sub, we identified the factors that were most important to determining detection efficiency under varying environmental conditions. The major determinant of detections of a transmitter was distance between receiver and transmitter (as expected and previously documented [14, 18-20]), with noise, angle between receiver and transmitter, WG heading and wave height also being important. These factors had varying importance depending on transmitter power output and receiver orientation (Additional file 1, [14]). Generally, distance, properties of seawater, sound frequency and background noise largely determine detection efficiencies 

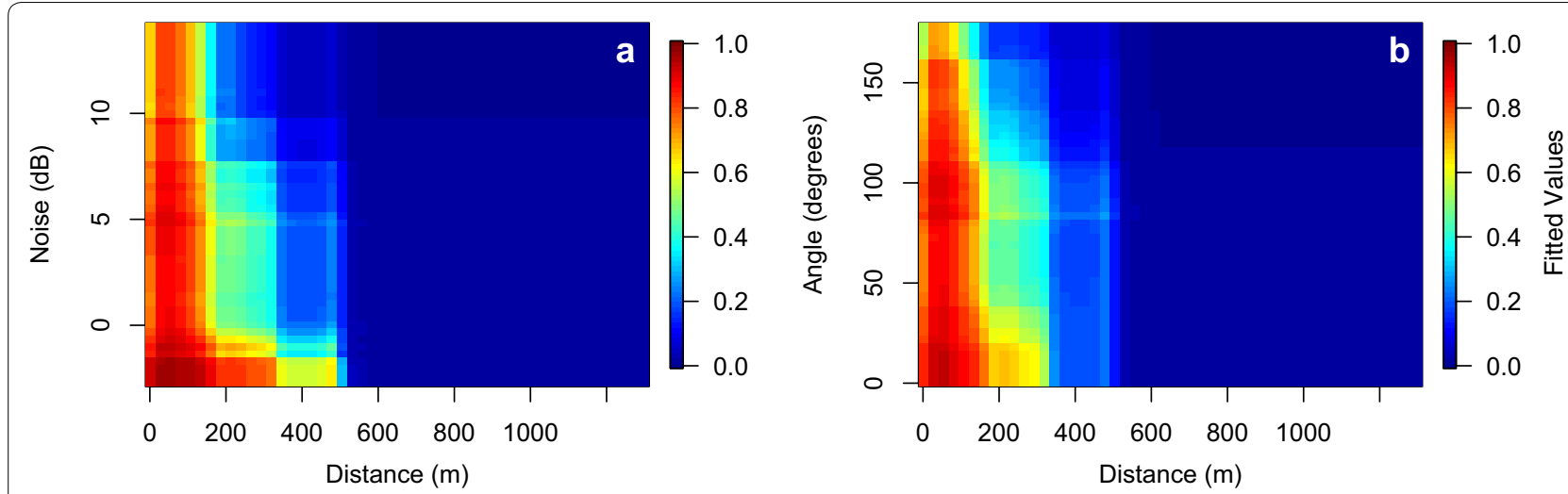

Fig. 7 Top two most frequent interactions in our models (see Additional file 2). a Interaction between distance (between the WG and mooring line) and noise, and $\mathbf{b}$ distance and angle (between the WG and mooring line). Fitted values are indicative of predicted probability of detections. These two examples are from model A, detections of the high transceiver on the WG's backward-facing transceiver. Visualizations of these interactions from other models were similar

[21]. As an acoustic signal travels through water, it is affected by spreading loss, refraction, scatter, absorption and attenuation $[21,22]$ and thus the distance a transmission travels depends on sound frequency of the signal, seawater composition and can be masked/distorted by background noise. Because the hydrophones are directional, orientation between the transmitters and receivers mattered. Thus, detections were more probable when the two were facing each other. Wind and surface waves can create additional background noise [23], and sub motions increase with larger waves and stronger winds; thus, these factors may decrease detection probabilities. Since both ambient noise and platform motion covary with sea state, it was difficult to determine the causal relationship of tag reception performance reductions within the scope of our research objectives. However, while the sources of ambient sound in the ocean are varied, we suspect the diel pattern in noise was from snapping shrimp that inhabits the region [24]. A full moon also occurred on January 31, but it is unclear whether biological signals (e.g., fish aggregations) commonly associated with full moon periods could be responsible for heightened noise levels on February 3 compared to February 7. The other predictor variables that were tested had a smaller contribution to determining the probability of detection, which included wind speed, sub pitch, roll and dynamics, temperature and mooring tilt. The weak effect of sub motion is promising for the approach, which is a similar to the minimal effects of vehicle attitude found for Slocum gliders [16]. Although our study was short (1 week), we suspect that if it was conducted under higher wind or wave conditions, vehicle factors such as pitch, roll and their rates could play a more important role in determining detection efficiencies. The conditions during the WG mission are common in southern California so the results reported here should be applicable to future surveys in the region.

Detection ranges varied by transmitter and receiver orientation, and power output. Our results revealed an external receiver mounted to the WG sub facing backward had nearly twice the detection range of a receiver facing forwards. The explained deviances of the backward-facing models (A-D) were also substantially higher than the forward-facing models $(E-H)$, suggesting other factors were affecting detection probabilities on the forward receiver. For example, hydrodynamic processes generated by forward motion likely influenced the detection range of the forward-facing receiver. In the lee of the sub, the water has likely already been perturbed by the subs wings and their motion and is homogenous enough to not interfere with the signal. With the superior backward-facing receiver orientation, a V7/V8 tag (at $142 \mathrm{~dB}$ ) would have a $20 \%$ detection efficiency to $\sim 0.15 \mathrm{~km}$; a V9 tag (at $148 \mathrm{~dB}$ ) would have a $20 \%$ detection efficiency to $\sim 0.2 \mathrm{~km}$; a V13 tag (at $153 \mathrm{~dB}$ ) had a $20 \%$ detection efficiency to $\sim 0.5 \mathrm{~km}$; and a V16 tag (at $154 / 160 \mathrm{~dB}$ ) would have a $20 \%$ detection efficiency to $\sim 0.3 / 0.8 \mathrm{~km}$ (Fig. 5, Table 1).

Detection ranges appeared to vary by study region. The detection efficiency was higher for the V13 tag compared to the high receiver, which had similar power outputs (154 dB vs. $153 \mathrm{~dB}$ ). The higher detection range of the V13 tag could be explained by the lower noise or shallower nearshore environment in the northern study region (Fig. 3a), or the tag being more efficient at emitting an omnidirectional signal compared to the larger and directional transceiver. It is also possible the high transceiver was affected by shading from the above medium and low transceiver, given its' lower detection efficiencies in all instances (Fig. 5a-c). Additionally, the missions 
Table 1 Comparison between transceiver power levels and power outputs of common VEMCO tag families. The V13-1H tag used in this study had an output power of $153 \mathrm{~dB}$

\begin{tabular}{lcl}
\hline Tag family & $\begin{array}{l}\text { Tag family power } \\
\text { output (dB re 1 uPa @ } \\
\mathbf{1 ~ m )}\end{array}$ & $\begin{array}{l}\text { Comparable transceiver } \\
\text { power level (dB re } \mathbf{1} \text { uPa } \\
\text { @ 1 m) }\end{array}$ \\
\hline V7 & 136 & Low (142) \\
V8 & $144-147$ & Low (142)/medium (148) \\
V9 & $145-151$ & Medium (148) \\
V13 & $147-153$ & Medium (148)/high (154) \\
V16 & $150-162$ & High (154)/very high (160) \\
\hline
\end{tabular}

differed between the two study regions where the northern WG path was rectangular and the southern was circular (Fig. 1), which results in different angles between the WG and mooring line.

One caveat to our study was the high probability of tag code collisions. This happens when multiple transmitters ping simultaneously, and overlapping transmissions likely decrease the receivers' ability to decode them (tag collision) even when within the detection range [21]. This was likely a problem in our study resulting in decreased detection efficiencies; therefore, detection probabilities are likely higher than reported here. Additionally, the increased detection efficiency of the V13 tag could be due to a lower probability of code collision as only two transmitters were pinging in the northern study region. Fortunately, close proximity detection interference $[16$, 25] does not appear to be an issue in our study, which occurs when the transmission power overwhelms the hydrophone. Slocum gliders with integrated VR2c receivers had this issue displaying low detection probability

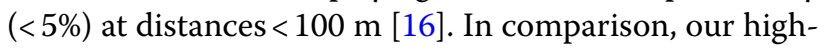
est detection efficiencies (50-95\%) were at this distance (Fig. 5).

Detection ranges varied by instrument setup but were overall comparable to other platforms. Interestingly, vehicle factors played a larger role in determining detection probabilities of the very high transceiver on mooring lines, which suggests detection efficiencies could be higher if this setup was tested in reverse with the very high transceiver on a mooring line instead of the WG sub. Our results suggested the mounting orientation of a receiver is critical to the success of hydrophone system and should be given thorough consideration and testing when using new platforms. Past studies using autonomous vehicles have had different mounting configurations, including horizontal VMTs or hydrophones facing forward or backward $[9,10,16]$, an upward and/ or downward facing VR2c with some on an angle $[6,7$, $10,16]$ or towing a VR2c that likely results in a variable orientation [15]. While most of these studies did not conduct a thorough range test, it is possible that receiver orientation in combination with different platform types (buoyancy driven, wave harnessing or propelled) could lead to different detection rates. While the overall $20 \%$ detection efficiencies in our study may seem low, the maximum detection range of all power outputs ranged from 0.5 to $1.2 \mathrm{~km}$. It is challenging to compare and contrast approaches as some studies only report detection efficiencies as a percentage and a distance while other studies provide bar plots and bin the data into different distance groupings. However, we found the detection ranges reported here were in line with other studies, including fixed arrays detecting similar tag types $(20 \%$ efficiency to $400 \mathrm{~m}$ of a $148 \mathrm{~dB}$ transmitter [17], 50\% efficiency to $183 \mathrm{~m}$ using V9 tags [26]), mobile animals (15\% efficiency to $400 \mathrm{~m}$ using VMTs at 146-149 $\mathrm{dB}$ [12]), autonomous gliders ( 20\% efficiency to $500-750 \mathrm{~m}$ using V16 tags [5], 20\% efficiency to $400 / 600 \mathrm{~m}$ for VMT/ VR2c using a VMT at $156 \mathrm{~dB}$ [16]) and WG with a towed receiver (estimated $500 \mathrm{~m}$ range for V13 tags [15]).

For future studies, the most suitable autonomous platform may depend on study region and timescale relevant to the research question. Propelled vehicles are capable of covering a large area in a short amount of time but have a short battery life (often $<1$ day) and may introduce more vehicle noise. They perform well in nearshore regions without a high risk of being swept onto shore or hitting the bottom. A propelled AUV was shown to be comparable or better than conventional boat-based tracking for both reference tags and tagged animals, possibly related to its ability to distance itself from surface noise [27]. Similar to propelled vehicles, WGs are capable of fine positional control but travel slower, can have long mission durations lasting $\sim 1$ year and likely produce lower platform noise. Buoyancy-driven vehicles (e.g., gliders) are the quietest, slowest and have the least amount of positional control but have a battery life of up to 3 months. When using autonomous vehicles, local knowledge is important for optimal mission planning and avoiding hazardous conditions (e.g., strong currents, steep bathymetry gradients, kelp forests) that could compromise route integrity [27]. In comparison, a stationary array can provide a long record ( $>1$ year) at one location without adding additional noise but is generally limited to shallow or nearshore environments where the instrument can be moored. All approaches are capable of relaying real-time detections, but this comes at different costs and levels of integration. All vehicles are capable of measuring many physical and biological environmental properties in addition to tag detections and are useful assets to consider in future telemetry work. Future studies may find a combination of approaches is most effective, which 
could include fixed coastal arrays, small vessel tracking, autonomous platforms and multiple tag types for information across many scales (e.g., [28-31]).

\section{Conclusion}

Our study suggests that WGs can be an effective biotelemetry asset that could supplement stationary arrays of acoustic receivers. Distance between the receiver and transmitter was the main factor influencing detection probability compared to environmental and vehicle factors. Detection efficiencies of animals may be even higher than reported here given the pitfalls of our study, including code collisions, mooring line setup that could have caused shading by the line or other receivers [32] or variations in receiver tilt on the mooring lines. Additionally, WG detection ranges could potentially be increased if the receiver was facing downward toward the seafloor or towed behind the vehicle, but further work is needed to quantify those ranges. For future studies limited to mounting one receiver on a WG sub, we suggest the receiver be mounted with the hydrophone facing backward. With the installation of a real-time telemetry component for the receiver on a WG, we envision realtime animal tracking and adaptive habitat mapping will occur. Multiple coordinated vehicles with receivers could also be very efficient at detecting animals within marine reserves or other areas of interest. We showed the utility of receivers on a WG in southern California and demonstrated that they can provide useful data without being fully integrated, a cost-effective approach. We anticipate WGs will become an important technology in tracking animal migrations and elucidating biophysical relationships.

\section{Methods}

In southern California, many fish inhabit nearshore coastal environments associated with rocky substrate or kelp forests. Animals of different sizes require different tag types and power outputs; thus, it is useful to compare multiple transmitter power settings, where a higher power transmission can be heard by receivers at a greater distance. We deployed two anchored mooring lines with surface floats, one nearshore in shallower water $(\sim 19 \mathrm{~m})$ in the north of our study region and the other further offshore in deeper water $(\sim 49 \mathrm{~m})$ in the south. Future Wave Glider (WG) missions to target coastal species would require WGs to transit parallel and as close as possible to the shoreline. The shallow mooring was a test of this type of mission while with the deep mooring we tested the detection range of different transmitter power outputs in all directions (Fig. 1).

\section{Wave Glider deployment}

We deployed a WG SV3 (Liquid Robotics, a Boeing company, Sunnyvale, CA) from February 2 to 9, 2018, off of La Jolla, CA (Fig. 1). A WG is a remotely piloted autonomous surface platform with a tether that connects a surface float to a subsurface glider (sub) with articulating wings (Fig. 2). WGs generate electrical power from solar panels in the float and harness wave energy for forward propulsion. The float also contains Iridium satellite communications and computer equipment, battery arrays and an Airmar Weather Station (Model PB200, Airmar Technology 166 Corporation, Milford, NH). The vehicle reported location and condition telemetry every $5 \mathrm{~min}$. We fabricated steel mounting brackets and plates for external sensor attachment. Attached to the WG sub were a stand-alone VR2W passive acoustic receiver (VEMCO Ltd., Nova Scotia, Canada) (facing forward), a VR2Tx acoustic transceiver (facing backward, set to transmit at a very high power level of $160 \mathrm{~dB}$ ) and accelerometer to measure pitch and roll (MAT-1 Data Loggers, Lowell Instruments, LLC, North Falmouth, MA, sampling at $2 \mathrm{~Hz}$ )(Fig. 2). A transceiver (VR2Tx) is a VR2W receiver with a built-in V16-like transmitter that also measured tilt, temperature and noise every $10 \mathrm{~min}$.

The WG was programmed to travel in an expanding box or circle-like pattern around two separate moorings. The surveys were not always symmetric around the moorings due to shallow bathymetry or other moored buoys in the region, which were avoided. The expanding circle around the south mooring had $50 \mathrm{~m}$ spacing from 50 to $700 \mathrm{~m}$ from the mooring and $100 \mathrm{~m}$ spacing from $700 \mathrm{~m}$ to $1 \mathrm{~km}$ (Fig. 1). The expanding boxes around the north mooring had $25 \mathrm{~m}$ spacing from 50 to $250 \mathrm{~m}, 50 \mathrm{~m}$ spacing from 250 to $500 \mathrm{~m}, 100 \mathrm{~m}$ spacing from 500 to $1000 \mathrm{~m}$ and $250 \mathrm{~m}$ spacing from 1 to $1.5 \mathrm{~km}$. The varying spacing was related to total duration of the mission (1 week) and estimated time it would take to complete the boxes given expected wave conditions (i.e., speed of the vehicle). During the mission, we also tested the performance of another sensor, which resulted in a few deviations from the southern mission track to transit the WG closer to shore during daytime.

\section{Stationary receivers and transmitters}

On the shallow mooring, an acoustic tag (V13-1H, VEMCO Ltd., $13 \mathrm{~mm}$ in diameter, $36 \mathrm{~mm}$ long, $6 \mathrm{~g}$ in seawater) was deployed at $7 \mathrm{~m}$ (vertically oriented using a zip tie to attach the tag $\sim 2$ inches away from mooring line; as suggested by VEMCO Ltd.) and a VR2W receiver at $9 \mathrm{~m}$ depth (Fig. 3). The tag transmitted uniquely coded acoustic signals every $20 \mathrm{~s}$ at a frequency of $69 \mathrm{kHz}$ and power level of $153 \mathrm{~dB}$ (re $1 \mu \mathrm{Pa}$ at $1 \mathrm{~m}$ ). The receiver detected each time the tag pinged. The tag/receiver was 
deployed mid water column as tagged species in the region utilize different depths, so we chose an intermediate depth as a proxy.

On the deep mooring, we deployed three VR2Tx transceivers. The transceivers were $1 \mathrm{~m}$ apart at 20,21 and $22 \mathrm{~m}$ depth and set to transmit at a low, medium and high power level (142 dB, $148 \mathrm{~dB}, 154 \mathrm{~dB}$ ), respectively (Fig. 3). No tilt information was obtained from the high transceiver due to a malfunction but we assumed the tilt was the same as the nearby transceivers. The transceivers transmitted at $90 \mathrm{~s}$ random delays. Due to code collisions, there was an $86 \%$ probability of detection (personal communication, VEMCO Ltd.). The power level of the transceivers is comparable to the output power of acoustic tags (Table 1). Therefore, while different sensors were attached to the two mooring lines, the V13 tag and high power transceiver had similar power output.

We used transceivers and tags with different power outputs to understand WG detection efficiencies of various tag types. Table 1 summarizes the common VEMCO tag families that correspond to the power outputs used here. Low, medium, high and very high power output could correspond to a V7/V8, V8/V9/V13, V13/V16 and V16 tags, respectively (Table 1). The V13 tag used in this study had an output power of $153 \mathrm{~dB}$, which is similar to a high power transceiver.

\section{Environmental and Wave Glider data}

At both mooring locations, we lowered a RBR concerto conductivity-temperature-depth (CTD) sensor (RBR Ltd. Canada) to measure the depth of the thermocline, which is known to influence acoustics. At the nearshore mooring, the CTD was lowered at 11:40 local time and at the offshore mooring, at 12:05 local time. Waves are also known to influence acoustics; thus, we obtained wave height data that were measured on a Scripps Pier buoy (Station 73), La Jolla, CA, by the Coastal Data Information Program (CDIP, http://cdip.ucsd. $\mathrm{edu} / \mathrm{sub}=$ data $\&$ nav $=$ historic $\&$ stn $=073 \&$ stream $=\mathrm{p} 1$ ).

Noise was measured on the transceivers in voltage. For interpretation, we converted the noise level on each transceiver to decibels, where $G_{\mathrm{dB}}=20 \log _{10}\left(V / V_{0}\right) . G_{\mathrm{dB}}$ is the power gain in $\mathrm{dB} . V$ is the measured voltage and $V_{0}$ is the average noise floor measured on each transceiver in a quiet room at night over $12 \mathrm{~h}$. All transceivers had a similar noise floor of $\sim 163 \mathrm{mV}$.

To determine the effect of receiver orientation on detection probability, an accelerometer was mounted beneath the WG tether on the sub (Fig. 2). We computed 1 -second averages of pitch and roll. We also determined the dynamics of the WG sub by calculating a 3-minute running mean and standard deviation of the pitch.
To determine the effect of distance on detection probability, we calculated the distance between each mooring line and the WG at all times. We determined the heading of the WG and the angle between the WG heading (i.e., receiver/transceiver) and each mooring line because we hypothesized that detections would be more likely if the receiver/transceiver was pointed toward the mooring line. All environmental variables that were not measured on a $1 \mathrm{~Hz}$ resolution were linearly interpolated.

\section{Data processing}

We removed all detections from tagged animals $(n=2)$ as well as false detections resulting from code collisions $(n=6)$. We determined whether each transmission from the tag or transceivers was recorded on the WG transceiver/receiver. Similarly, we also determined whether transmissions from the WG transceiver were recorded on the mooring line transceivers/receiver. To do this, we used the times of each tag/transceiver transmission, which was recorded on each respective transceiver or receiver. For all transmissions, we determined whether they were received (yes or no) on each transceiver/receiver of interest. Occasionally, a small time gap between the transmission and detection was present, likely related to a very small (seconds) time drift. For the north mission, the tag transmitted every 23-24 s to be exact. A transmission was considered to be detected if it was recorded within $\pm 4 \mathrm{~s}$ of the transmission, which was the largest gap between a transmission and detection; this offset was small enough to prevent false positives. Similarly, on the transceivers, the smallest time between pings was $63 \mathrm{~s}$. We used $\mathrm{a} \pm 7 \mathrm{~s}$ range to determine whether a transmission was detected, which was the largest gap recorded between these transmissions and detections.

We merged detection information on whether each emitted acoustic signal was heard (binary, yes or no) to all environment conditions (wind, waves, water temperature, noise), WG factors (pitch, roll and their rates of the sub; heading, angle between the WG and mooring line), transceiver factors (tilt) and distance between the WG and mooring line. It should be noted that all instrument clocks were synced prior to deployment. Given the short mission duration (1 week), we assumed very little time drift occurred and therefore, believed instantaneous measurements from independent sensors corresponded to roughly the correct time.

To determine the predictor variable that influenced detection efficiency, we modeled the binomial presence/ absence of detections on the forward- and backwardfacing receiver/transceiver on the WG for the V13 tag, high, medium and low transceiver (see Table 2 for models). We also modeled the presence/absence of detections 
Table 2 Boosted regression tree (BRT) models to understand the factors that determine whether each acoustic ping was detected (yes/no) on each receiver or transceiver

\begin{tabular}{|c|c|c|c|}
\hline & Model & $\begin{array}{l}\text { Explained } \\
\text { deviance } \\
(\%)\end{array}$ & AUC \\
\hline \multicolumn{4}{|c|}{ Detections on the WG backward-facing transceiver of } \\
\hline (A) High transceiver & \multirow{3}{*}{$\begin{array}{l}\sim \text { wind.speed + wave.height + sub.pitch + sub.roll + WG.heading + WG.angle + Distance + mooring. } \\
\text { noise + mooring.tilt + mooring.temp + sub.dynamics }\end{array}$} & 39.93 & 0.92 \\
\hline (B) Medium transceiver & & 49.35 & 0.94 \\
\hline (C) Low transceiver & & 53.29 & 0.96 \\
\hline (D) V13 tag & $\begin{array}{l}\sim \text { wind.speed + wave.height + sub.pitch + sub.roll +WG.heading + WG.angle + Distance +WG. } \\
\text { temp + sub.dynamics }\end{array}$ & 63.99 & 0.97 \\
\hline \multicolumn{4}{|c|}{ Detections on the WG forward-facing transceiver of } \\
\hline (E) High transceiver & \multirow{3}{*}{$\begin{array}{l}\sim \text { wind.speed + wave.height + sub.pitch + sub.roll + WG.heading + WG.angle + Distance + mooring. } \\
\text { noise + mooring.tilt + mooring.temp + sub.dynamics }\end{array}$} & 28.11 & 0.89 \\
\hline (F) Medium transceiver & & 43.01 & 0.94 \\
\hline (G) Low transceiver & & 48.33 & 0.95 \\
\hline (H) V13 tag & $\begin{array}{l}\sim \text { wind.speed + wave.height + sub.pitch + sub.roll +WG.heading + WG.angle + Distance +WG. } \\
\text { temp + sub.dynamics }\end{array}$ & 37.23 & 0.90 \\
\hline \multicolumn{4}{|c|}{ Detections of the very high transceiver (backward facing) on WG by } \\
\hline (I) High transceiver & \multirow{3}{*}{$\begin{array}{l}\sim \text { wind.speed + wave.height + sub.pitch + sub.roll + WG.heading + WG.angle + Distance + sub. } \\
\text { noise + mooring.tilt + mooring.temp + sub.dynamics }\end{array}$} & 30.03 & 0.86 \\
\hline (J) Medium transceiver & & 23.61 & 0.82 \\
\hline (K) Low transceiver & & 24.30 & 0.82 \\
\hline (L) VR2W & $\begin{array}{l}\sim \text { wind.speed + wave.height + sub.pitch + sub.roll + WG.heading + WG.angle + Distance + sub. } \\
\text { noise + WG.temp + sub.dynamics }\end{array}$ & 32.50 & 0.85 \\
\hline
\end{tabular}

The north mooring had a VR2W receiver (not transceiver) so noise, tilt and temperature data were not available for that mooring line region; therefore, temperature measured on the Wave Glider (WG) transceiver was used instead

wind.speed is the wind speed measured by the WG. wave.height was measured on the CDIP buoy. sub.pitch and sub.roll are the pitch and roll of the WG sub measured by the accelerometer. sub.dynamics is the running 3-minute standard deviation in sub pitch. sub.noise is the noise measured by the WG transceiver. WG.heading is the heading of the WG. WG.angle is the angle between the WG and the respective mooring line. Distance is the distance between the WG and the respective mooring line. mooring.noise, mooring.tilt and mooring.temp are the noise, tilt and temperature, respectively, measured by the mooring line transceivers

on the mooring line transceivers/receivers from the WG transceiver. We limited the data in the analysis to times when the distance between the WG and mooring line was $<1.3 \mathrm{~km}$, which was $100 \mathrm{~m}$ beyond the furthest detection. We used a boosted regression tree (BRT) modeling approach, which is popular machine learning technique that outperforms traditional approaches and can elucidate complex nonlinear relationships [33-36]. The approach can handle outliers, irrelevant predictors, missing data, multicollinearity and violations of traditional statistical assumptions, which include independent and unequal variances [34, 37]. Many simple models (each classification tree) are iteratively fit to a random subset of the data (bag fraction) that includes stochasticity and are combined to estimate a response [33, 38].

We used establishing protocols $[34,37]$ and the brt. functions package in $\mathrm{R}$ [34] ( $\mathrm{R}$ Development Core Team 2017). We used a Bernoulli (binomial) distribution, a tree complexity of 3 (number of nodes controlling which interactions are fitted) as higher orders are likely unnecessary $[39,40]$ and a bag fraction of $0.7[34$, 39]. The learning rate (contribution of successive trees to the growing model) was tuned so that at least 1000 trees were included in the final model (following methods outlined in [34]). We explored models using a range of learning rates $(0.05,0.01,0.005,0.001,0.0005)$ and selected the optimal rate based on the lower predictive deviance to avoid overfitting [34]. In addition, to determine the robustness of the model, we used fivefold crossvalidation where $75 \%$ of the data were used for training and $25 \%$ for testing, quantified the relative influence of predictor variables and used partial dependency plots to reveal the influence of a variable after accounting for the average effects of all other variables in the model [34, 41]. Partial plots may not show the comprehensive effects of a variable on the response, especially if strong interactions between predictor variables were present. Model accuracy was determined using the area under the receiver operating curve (AUC) measurement, where a perfect performance would have an AUC of 1 [42]. The proportion of deviance explained was also reported.

\section{Additional files}

Additional file 1. Variable percent contribution.

Additional file 2. Partial dependence plots.

Additional file 3. Two-way variable interactions. 


\section{Abbreviations}

WG: Wave Glider; AUV: autonomous underwater vehicle; VR2c: VEMCO cabled receivers; VMT: VEMCO mobile transceivers; BRT: boosted regression tree; AUC : area under the curve.

\section{Authors' contributions}

MAC and ET designed the study, and MAC led mission programming and deployment. MAC and MC analyzed Wave Glider and received the data, SM analyzed the accelerometry data and MAC ran the statistical models. MAC wrote the initial manuscript. All authors read and approved the final manuscript.

\section{Author details}

${ }^{1}$ Scripps Institution of Oceanography, University of California at San Diego, La Jolla, CA 92093, USA. ${ }^{2}$ Institute of Marine Science, University of California Santa Cruz, SantaCruz, CA 95064, USA.

\section{Acknowledgements}

We thank the staff at the Coastal Observing Research and Development Center at the Scripps Institution of Oceanography, especially Nixon Carruthers, Daniel Bedenko and Michael Jilka for help in Wave Glider setup, mission programming and deployment/recovery. We thank Brice Semmens for lending us two VEMCO receivers and Christopher Lowe for his input on mission planning. We also thank Matthew Holland at VEMCO for his advice throughout the project.

\section{Competing interests}

The authors declare that they have no competing interests.

\section{Availability of data and materials}

The datasets used and/or analyzed during the current study are available from the corresponding author on reasonable request.

\section{Consent for publication}

Not applicable.

\section{Ethics approval and consent to participate}

Not applicable.

\section{Funding}

None.

\section{Publisher's Note}

Springer Nature remains neutral with regard to jurisdictional claims in published maps and institutional affiliations.

Received: 28 September 2018 Accepted: 8 November 2018 Published online: 03 December 2018

\section{References}

1. Hussey NE, Kessel ST, Aarestrup K, et al. Aquatic animal telemetry: a panoramic window into the underwater world. Science. 2015;348:1255642.

2. Kessel ST, Cooke SJ, Heupel MR, et al. A review of detection range testing in aquatic passive acoustic telemetry studies. Rev Fish Biol Fish. 2014:24:199-218.

3. Oliver MJ, Breece MW, Fox DA, et al. Shrinking the haystack: using an AUV in an integrated ocean observatory to map Atlantic Sturgeon in the coastal ocean. Fisheries. 2013;38:210-6.

4. Breece MW, Fox DA, Dunton KJ, et al. Dynamic seascapes predict the marine occurrence of an endangered species: Atlantic Sturgeon Acipenser oxyrinchus oxyrinchus. Methods Ecol Evol. 2016;7:725-33.

5. Haulsee DE, Breece MW, Miller DC, Wetherbee BM, Fox DA, Oliver MJ. Habitat selection of a coastal shark species estimated from an autonomous underwater vehicle. Mar Ecol Prog Ser. 2015;528:277-88.

6. Clark CM, Forney C, Manii E, et al. Tracking and following a tagged leopard shark with an autonomous underwater vehicle. J Field Robot. 2013;30:309-22.
7. Lin Y, Hsiung J, Piersall R, White C, Lowe CG, Clark CM. A multi-autonomous underwater vehicle system for autonomous tracking of marine life. J Field Robot. 2017:34:757-74.

8. Grothus TM, Dobarro J, Ladd J, et al. Use of a multi-sensored AUV to telemeter tagged Atlantic sturgeon and map their spawning habitat in the Hudson River, USA. In: Autonomous underwater vehicles, 2008. AUV 2008. IEEE/OES; 2008; IEEE; 2008. https://doi.org/10.1109/AUV.2008.53475 97.

9. Grothues TM, Dobarro J, Eiler J. Collecting, interpreting, and merging fish telemetry data from an AUV: remote sensing from an already remote platform. Auton Underw Veh (AUV). 2010;2010:1-9.

10. Carlon R. Tracking tagged fish using a wave glider. In: OCEANS'15 MTS/ IEEE Washington; 2015; IEEE; 2015. https://doi.org/10.23919/OCEAN S.2015.7404617.

11. How JR, de Lestang S. Acoustic tracking: issues affecting design, analysis and interpretation of data from movement studies. Mar Freshw Res. 2012;63:312-24.

12. Baker $\mathrm{LL}$, Jonsen ID, Flemming JEM, et al. Probability of detecting marine predator-prey and species interactions using novel hybrid acoustic transmitter-receiver tags. PLoS ONE. 2014:9:e98117.

13. Mathies NH, Ogburn MB, McFall G, Fangman S. Environmental interference factors affecting detection range in acoustic telemetry studies using fixed receiver arrays. Mar Ecol Prog Ser. 2014;495:27-38.

14. Melnychuk M. Detection efficiency in telemetry studies: definitions and evaluation methods. Telemetry techniques: a user guide for fisheries research American Fisheries Society, Bethesda, Maryland. 2012339-357.

15. Cote D, Nicolas J-M, Whoriskey FG, et al. Characterizing snow crab (Chionoecetes opilio) movements in the Sydney Bight (Nova Scotia, Canada): a collaborative approach using multi-scale acoustic telemetry. Can J Fish Aquat Sci. 2018;999:1-13

16. Oliver MJ, Breece MW, Haulsee DE, et al. Factors affecting detection efficiency of mobile telemetry Slocum gliders. Anim Biotelem. 2017;5:14.

17. Reubens J, Verhelst P, van der Knaap I, Deneudt K, Moens T, Hernandez F. Environmental factors influence the detection probability in acoustic telemetry in a marine environment: results from a new setup. Hydrobiologia. 2018; https://doi.org/10.1007/s10750-017-3478-7.

18. Topping DT, Szedlmayer ST. Home range and movement patterns of red snapper (Lutjanus campechanus) on artificial reefs. Fish Res. 2011;112:77-84.

19. How JR, de Lestang S. Acoustic tracking: issues affecting design, analysis and interpretation of data from movement studies. Mar Freshw Res. 2012:63:312-24.

20. Welsh JQ, Fox RJ, Webber DM, Bellwood DR. Performance of remote acoustic receivers within a coral reef habitat: implications for array design. Coral Reefs. 2012;31:693-702.

21. Heupel MR, Semmens JM, Hobday AJ. Automated acoustic tracking of aquatic animals: scales, design and deployment of listening station arrays. Mar Freshw Res. 2006:57:1-13.

22. Singh L, Downey NJ, Roberts MJ, et al. Design and calibration of an acoustic telemetry system subject to upwelling events. Afr J Mar Sci. 2009:31:355-64.

23. Gjelland $K \varnothing$, Hedger RD. Environmental influence on transmitter detection probability in biotelemetry: developing a general model of acoustic transmission. Methods Ecol Evol. 2013;4:665-74.

24. Everest FA, Young RW, Johnson MW. Acoustical characteristics of noise produced by snapping shrimp. J Acoust Soc Am. 1948;20:137-42.

25. Kessel ST, Hussey NE, Webber DM, et al. Close proximity detection interference with acoustic telemetry: the importance of considering tag power output in low ambient noise environments. Anim Biotelem. 2015;3:5.

26. TinHan TC, Mohan JA, Dumesnil M, DeAngelis BM, Wells RJD. Linking habitat use and trophic ecology of spotted seatrout (Cynoscion nebulosus) on a restored oyster reef in a subtropical estuary. Estuaries Coast. 2018:41:1-13.

27. Eiler JH, Grothues TM, Dobarro JA, Masuda MM. Comparing autonomous underwater vehicle (AUV) and vessel-based tracking performance for locating acoustically tagged fish. Mar Fish Rev. 2013;75:27-42.

28. Voegeli FA, Smale MJ, Webber DM, Andrade Y, O'dor RK. Ultrasonic telemetry, tracking and automated monitoring technology for sharks. Environ Biol Fishes. 2001;60:267-82. 
29. Welch DW, Boehlert GW, Ward BR. POST—-the Pacific Ocean salmon tracking project. Oceanol Acta. 2002;25:243-53.

30. Holland K, Brill R, Ferguson S, Chang R, Yost R. A small vessel technique for tracking pelagic fish. Mar Fish Rev. 1985;47:26-32.

31. $\mathrm{Ng} \mathrm{CL}$, Able KW, Grothues TM. Habitat use, site fidelity, and movement of adult striped bass in a southern New Jersey estuary based on mobile acoustic telemetry. Trans Am Fish Soc. 2007;136:1344-55.

32. Clements S, Jepsen D, Karnowski M, Schreck CB. Optimization of an acoustic telemetry array for detecting transmitter-implanted fish. N Am J Fish Manag. 2005;25:429-36.

33. De'Ath G. Boosted trees for ecological modeling and prediction. Ecology. 2007;88:243-51.

34. Elith J, Leathwick JR, Hastie T. A working guide to boosted regression trees. J Anim Ecol. 2008;77:802-13.

35. Buston PM, Elith J. Determinants of reproductive success in dominant pairs of clownfish: a boosted regression tree analysis. J Anim Ecol. 2011;80:528-38.

36. Oppel S, Meirinho A, Ramírez I, et al. Comparison of five modelling techniques to predict the spatial distribution and abundance of seabirds. Biol Cons. 2012;156:94-104.
37. Leathwick JR, Elith J, Francis MP, Hastie T, Taylor P. Variation in demersal fish species richness in the oceans surrounding New Zealand: an analysis using boosted regression trees. Mar Ecol Prog Ser. 2006;321:267-81.

38. Friedman JH. Greedy function approximation: a gradient boosting machine. Ann Stat. 2001;29:1189-232.

39. Scales KL, Hazen EL, Maxwell SM, et al. Fit to predict? Eco-informatics for predicting the catchability of a pelagic fish in near real time. Ecol Appl. 2017;27:2313-29

40. Soykan CU, Eguchi T, Kohin S, Dewar H. Prediction of fishing effort distributions using boosted regression trees. Ecol Appl. 2014;24:71-83.

41. Hastie T, Tibshirani R, Friedman J. The elements of statistical learning. New York: Springer; 2001.

42. Hosmer DW, Lemeshow S. Applied logistic regression. 2nd edn:Wiley;2000
Ready to submit your research? Choose BMC and benefit from:

- fast, convenient online submission

- thorough peer review by experienced researchers in your field

- rapid publication on acceptance

- support for research data, including large and complex data types

- gold Open Access which fosters wider collaboration and increased citations

- maximum visibility for your research: over $100 \mathrm{M}$ website views per year

At $\mathrm{BMC}$, research is always in progress.

Learn more biomedcentral.com/submissions 